\title{
On the Group Delay Statistics of Few-Mode Fibres with Intermediate Linear Mode Coupling
}

\author{
Filipe M. Ferreira, Naoise Mac Suibhne, Christian S. Costa, Mariia Sorokina, Stylianos Sygletos, Andrew Ellis \\ Aston Institute of Photonic Technologies, Aston University, Birmingham, B4 7ET, UK \\ f.ferreira@aston.ac.uk
}

\begin{abstract}
We report an investigation on the statistics of group delay for few-mode fibres operating in the weak and strong linear coupling regimes as well as in the intermediate coupling regime. A single expression linking the standard deviation of the group delay spread to the fibre linear mode coupling is validated for any coupling regime, considering up to six linearly polarized guided modes. Furthermore, the study of the probability density function of the group delays allowed deriving and validating an analytical estimation for the maximum group delay spread as a function of linear mode coupling.
\end{abstract}

\section{INTRODUCTION}

Mode-division multiplexing over few-mode fibres (FMFs) has been proposed as a next-generation solution to overcome the impeding installed capacity exhaustion of current single-mode fibres (SMFs). However, MDM-FMF systems require significantly higher equalizer complexity given the overall group-delay (GD) spread due to differential mode delay and linear mode coupling [1], [2]. Thereby, the estimation of the maximum GD spread is of foremost importance. However, most studies on the statistical properties of the GDs consider the two extreme coupling regimes, weak and strong [1], [2]. Recently, we validated one single expression linking the standard deviation of GDs to the coupling strength for any coupling regime, considering a FMF guiding 3 linearly polarized (LP) modes [3] and a FMF guiding 6 LP modes [4]. More recently, in [5], this expression was rederived analytically.

In this paper, we review and extend our previous studies [3], [4]. The standard deviation and probability density function of the coupled GDs as well as the cumulative distribution of the maximum GD spread are investigate for the intermediate coupling regime. Furthermore, analytical estimators for these quantities are validated.

\section{Group-Delay Statistics}

The statistical theory of polarization mode dispersion (PMD) in SMFs [6] has identified two coupling regimes, the strong coupling and the weak coupling, determined by one parameter, the correlation length $L_{c}$. The $L_{c}$ is defined as the length for which the average power in the orthogonal polarization is within $e^{-2}$ of the power in the starting polarization. When the transmission distance $L$ is much bigger than $L_{c}$, the fibre is in the strong coupling regime, conversely, when the $L$ is much smaller than the $L_{c}$, the fibre is in the weak coupling regime. Furthermore, the PMD theory predicts the existence of principal states of polarization (PSPs) with well-defined group delays (GDs). In the weak coupling regime, the spread of the uncoupled GDs scales linearly with $L$, and in the strong coupling regime, the spread of the coupled GDs of the PSPs scales with $L^{1 / 2}$. In [7], an elegant expression linking the standard deviation (STD) of the coupled GDs of the PSPs $\left(\sigma_{g d}\right)$ to the fibre correlation length was presented and was shown to be valid for both coupling regimes and also for the transition region between them:

$$
\sigma_{g d}=\sqrt{2} \sigma_{\tau} L_{c}\left[\exp \left(-L / L_{c}\right)+L / L_{c}-1\right]^{1 / 2}
$$

where $\sigma_{\tau}$ is the STD of the uncoupled GDs per unit length.

In [8], it has been shown that the PMD theory can be extend to the multimode case, where coupled modes having well defined GDs were called principal modes (PMs). Neglecting the mode dependent loss, the linear propagation through a FMF guiding $N$ modes can be described by a $N \times N$ unitary matrix, $\mathbf{M}(\omega)$, where $\omega$ is the optical angular frequency. In this case, the GDs of the PMs, at each single frequency, are the eigenvalues of the Hermitian GD operator $\mathbf{G}(\omega)=\mathbf{j} \mathbf{M}_{\omega}(\omega) \mathbf{M}^{H}(\omega)$, where $\mathbf{M}_{\omega}(\omega)=d \mathbf{M}(\omega) / d \omega$ and ${ }^{H}$ stands for Hermitian transpose. Finally, the STD of the coupled GDs of the PMs of a link with $K$ sections $\left(\sigma_{g d}\right)$ can be expressed as a function of the STD of the uncoupled GDs of the PMs along one section $\left(\sigma_{\tau, \text { section }}\right)$, assuming that all fibre sections have identical statistical properties. In the strong coupling regime, the fibre can be modelled as $K$ independent sections with length approximately equal to or slightly greater than $L_{c}$, where the coupling is introduced in each section by a random unitary matrix. The STD of the coupled PMs of a link operating in this regime is $\sigma_{g d}=K^{1 / 2} \cdot \sigma_{\tau, \text { section }}$. In the weak coupling regime, the mode coupling can be neglected, and the STD of the uncoupled PMs is given by $\sigma_{g d}=K \cdot \sigma_{\tau, \text { section. }}$. Furthermore, with intermediate coupling, it has been shown in [5] that $\sigma_{g d}$ in FMFs also follow (1).

\section{Linear Mode Coupling}

The mode coupling is quantified with the parameter $X T_{m}=\sum_{v \neq \mathrm{LP} 01}\left(P_{v} / P_{m}\right)$, where $P_{v}$ is the power of mode $v$, after a given fibre segment under test, when only the $m$ mode was launched, where $m$ is the mode that shows higher 


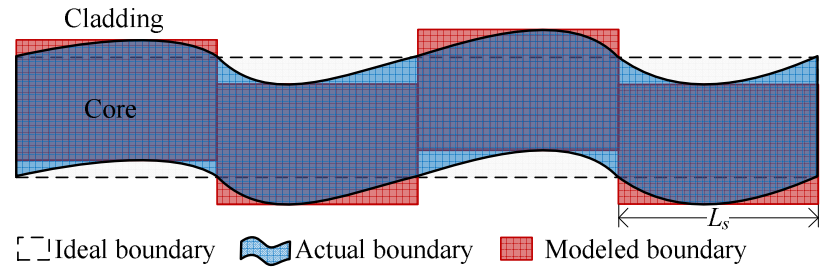

Fig. 1. Sketch of a fibre with distorted core-cladding boundary.

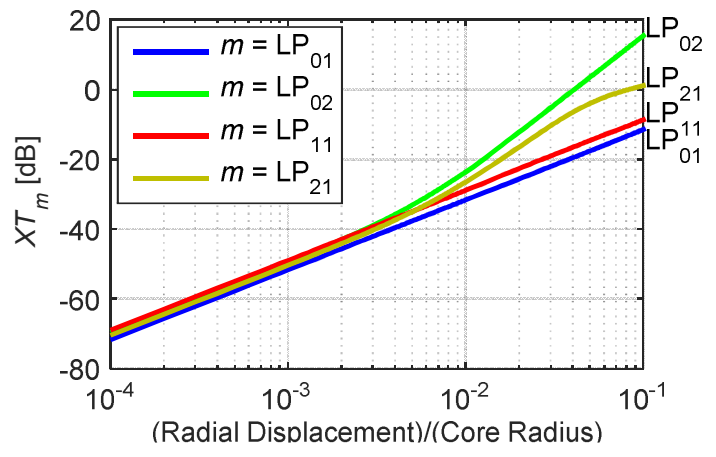

Fig. 2. $X T_{m}$ averaged over the azimuth displacement as a function of the radial displacement

coupling strength. In the FMF case, we generalize $L_{c}$ as the length for which $\left(P_{m}-\sum_{v \neq m} P_{v}\right)=e^{-2}$, equivalent to $X T_{m}=\left[e^{2}-1\right] /\left[e^{2}+1\right](-1.18 \mathrm{~dB})$. In the literature, the mode coupling values of fabricated FMFs range from $-50 \mathrm{~dB} / 100 \mathrm{~m}$ to $-40 \mathrm{~dB} / 100 \mathrm{~m}$ for fibres with step-index or graded-index profiles [9],[10] $\left(L_{c}\right.$ values ranging from 7,000 to $800 \mathrm{~km}$ ), going up to $-28 \mathrm{~dB} / 100 \mathrm{~m}$ for coupled multi-core fibres [11] and $-7 \mathrm{~dB} / 100 \mathrm{~m}$ for fibres with ring-index profiles [12]. Note that for a fibre with $L_{c}=800 \mathrm{~km}$, the strong coupling regime is fully achieved only for $L>8,000 \mathrm{~km}\left(L \gg L_{c}\right)$. Consequently, for typical fibre link distances, less than $1000 \mathrm{~km}$, the system is neither operating in the strong coupling regime nor the weak coupling regime $\left(L \sim L_{c}\right)$. Thus, the performance of FMF systems, for these mode coupling levels and typical distances, might not be accurately modelled by random unitary matrices (every $\sim 100 \mathrm{~m}$ ) because the impact of group delay spread and intermodal nonlinearity on the performance might be underestimated. In the next section, the impact of the coupling strength on the GD spread is accessed as well as the average $X T$ per km required for strong coupling operation.

Firstly we briefly review the mode coupling model [13]. We model the fibre imperfections responsible for mode coupling as random fluctuations of the core centre position, given by: $\varepsilon(x, y, z)=\varepsilon_{p}(x+\delta x(\mathrm{z}), y+\delta y(\mathrm{z}), z)$, where $\varepsilon$ is the perturbed permittivity, $\varepsilon_{p}$ is the ideal relative permittivity, $\delta x$ and $\delta y$ are the random displacement of the abscissa and ordinate coordinates, respectively. The proposed model divides the fibre in multiple sections of length $L_{s}$, each with a constant random displacement of the core centre position, as shown in Fig. 1. Therefore, each section has constant coupling coefficients. In order to analytically describe the coupling arising from the waveguide imperfections, we used the coupled wave theory [14], particularly the following coupled-mode equations:

$$
\begin{gathered}
\partial_{z} \tilde{A}_{\mu}(z, \omega)=-\mathrm{j}\left[\beta_{\mu}(\omega)-\beta_{0 \mu}\right] \tilde{A}_{\mu}(z, \omega)-\mathrm{j} \sum_{v \neq \mu} C_{\mu v}(z) \tilde{A}_{\nu}(z, \omega) \exp \left[\mathrm{j}\left(\beta_{0 \mu}-\beta_{0 v}\right) z\right] \\
C_{\mu v}(z)=\frac{\omega \varepsilon_{0}}{4} \iint_{-\infty}^{+\infty}\left[\varepsilon(x, y, z)-\varepsilon_{p}(x, y, z)\right] \mathbf{E}_{\mu} \cdot \mathbf{E}_{v}^{*} d x d y
\end{gathered}
$$

where $\tilde{A}_{\mu}(z, \omega)$ is the Fourier transform of the mode $\mu$ slowly varying field envelope $A_{\mu}, \beta_{\mu}(\omega)$ is the mode $\mu$ propagation constant, and $\beta_{l \mu}$ is the $l^{\text {th }}$ order coefficient of a Taylor expansion of $\beta_{\mu}(\omega)$ centred at the carrier frequency $\omega_{0} . C_{\mu \nu}$ are the coupling coefficients given by the area integral of the dot product of the electrical fields of mode $\mu$ and mode $v$, over the area where the permittivity difference is nonzero. Finally, the unitary matrix $\mathbf{M}(\omega)$ for a FMF can be obtained by numerically solving (2) or by using the semi-analytical solutions presented in [15].

Fig. 2 shows the mode coupling strength averaged over the azimuth displacement, as a function of the normalised radial displacement, for a 6 LP mode fibre presented in [16]. Note that, coupling strengths are calculated considering degenerate modes such as $\mathrm{LP}_{11 \mathrm{a}}$ and $\mathrm{LP}_{11 \mathrm{~b}}$ as one mode. In Fig. 2, it can be seen that the mode coupling strength only depends significantly on the mode being considered for displacements higher than $1 \%$. Such higher coupling for $\mathrm{LP}_{02}$ and $\mathrm{LP}_{21}$ can be explained noting they belong to the same LP mode group. Moreover, $X T_{L P_{2 l}}<X T_{L P_{02}}$ for any displacement in Fig. 2 because any power launched in $L_{2} P_{21}$ couples preferentially with $\mathrm{LP}_{21 \mathrm{~b}}$ (and vice-versa) and in the second place to $\mathrm{LP}_{02}$. Given the higher values of $X T_{L P_{02}}$, we define $L_{c}$ for this mode. Note that $X T_{m}$ values above $10 \mathrm{~dB}$ mean that almost all power launched in mode $m$ has been transferred to other modes.

\section{Results}

In this section, we evaluate the statistics of the GDs for a FMF guiding 6 modes and presenting a DMD of $5.19 \mathrm{ps} / \mathrm{km}$ (we assumed zero DMD between degenerate LP modes) [16]. The $X T_{L P_{02}}$ value was varied from -50 to $0 \mathrm{~dB} / 100 \mathrm{~m}$ by varying the amplitude of the variation in lateral section offset, assuming a section length of $100 \mathrm{~m}$. This range fully covers the range of coupling values presented in the literature [9]-[12]. Finally, the GDs were calculated through direct numerical solution of the coupled-mode equations describing the mode coupling [13], [15].

Fig. 3 shows the standard deviation of the GDs of the PMs $\left(\sigma_{g d}\right)$ as a function of distance up to $10,000 \mathrm{~km}$, obtained by averaging over 2000 different realizations of lateral offsets giving rise to a given $X T_{L P 02}$ value. As was 


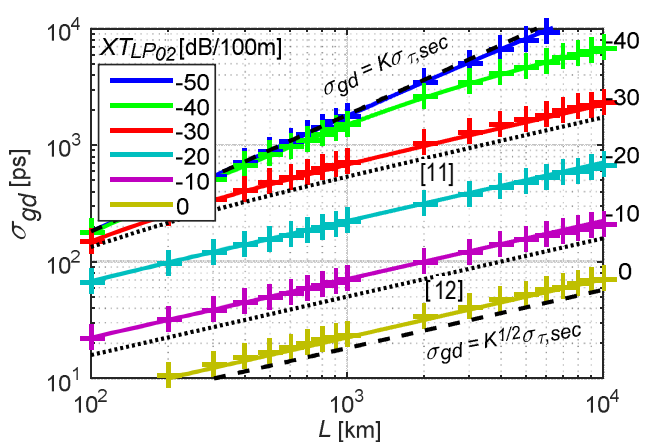

Fig. 3. Standard deviation of the GDs of the PMs as a function of transmission distance showing simulation results (markers) and analytical results (solid lines).

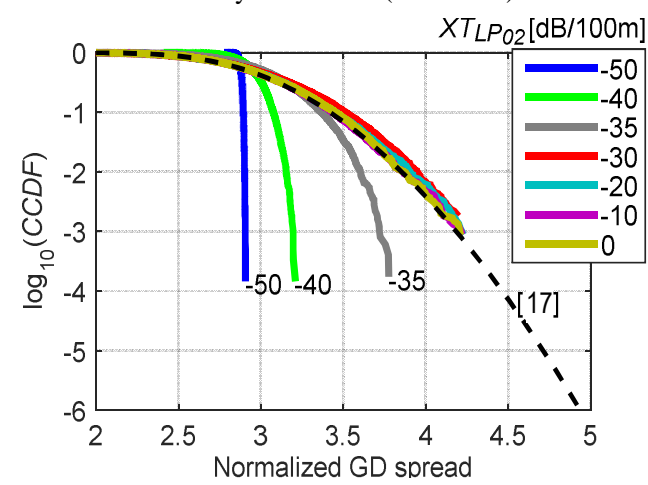

Fig. 5. Complementary cumulative distribution of the normalized GD spread, obtained through simulation after $1000 \mathrm{~km}$, with different $X T_{L P_{02}}$ values.

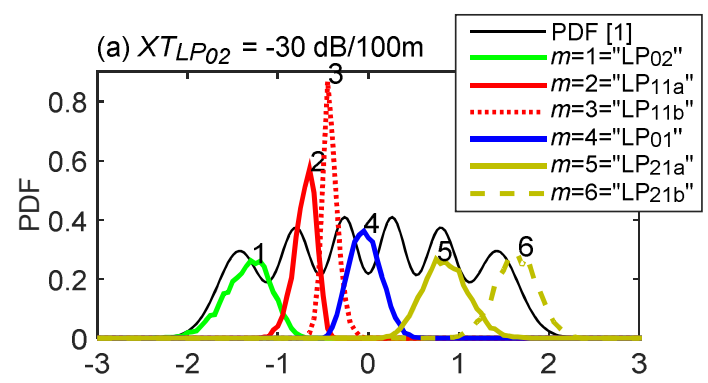

(b) $X T_{L P_{02}}=-20 \mathrm{~dB} / 100 \mathrm{~m}$

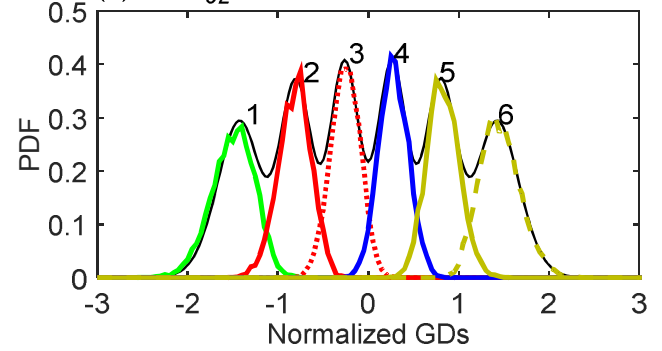

Fig. 4. Probability density function of the ordered normalized GDs $\left(\tau_{m} / \sigma_{g d}\right)$, obtained through simulation after $1000 \mathrm{~km}$, with different $X T_{L P_{02}}$ values.

found for 3 LP modes [3], Fig. 3 shows a good agreement between numerical simulation and the analytical expression (1), for any coupling value studied and for any distance up to $10,000 \mathrm{~km}$. This verifies that Eq. (1) remains valid for 6-mode fibres (the validity for 3-mode fibres had been studied in [3]). In Fig. 3, it can be seen that for coupling values ranging from -50 to $-40 \mathrm{~dB} / 100 \mathrm{~m}, \sigma_{g d}$ scales approximately linearly with distance. But, at $-40 \mathrm{~dB} / 100 \mathrm{~m}$ the deviation from linear growth is already noticeable above $1,000 \mathrm{~km}$, thus even with such a low coupling, the FMF is operating in intermediate coupling regime. Increasing $X T_{L P_{02}}, \sigma_{g d}$ gradually converges to the strong coupling regime. However, even for a $X T_{L_{02}}$ equal to $-7 \mathrm{~dB} / 100 \mathrm{~m}$ (the highest value found in literature [12]) the fibre is still not fully operating in the strong coupling regime when considering a section length of $100 \mathrm{~m}$. In this case, assuming strong coupling regime (random unitary matrices every $100 \mathrm{~m}$ ), would underestimated $\sigma_{g d}$ by a factor of 2.76 .

Fig. 4 shows the PDF of the ordered GDs $\left(\tau_{m}, \tau_{1} \leq \tau_{2} \leq \cdots \leq \tau_{6}\right)$, normalized by the $\sigma_{g d}$ of the PMs, after $1000 \mathrm{~km}$ for two different coupling values, overlapped with the analytical marginal PDF (thin black line) derived for the strong coupling regime [1]. Note that the normalization factor $\left(\sigma_{g d}\right)$ depends on the $X T_{L P_{02}}\left(L_{c}\right)$ value, see Eq. (1). Fig. 4 (a) shows that for $-30 \mathrm{~dB} / 100 \mathrm{~m}$ the GDs of the PMs vaguelly resemble the GDs of the LP modes given the impulse-like PDF of $\tau_{2}$ ("LP ${ }_{11 a}$ ") and $\tau_{3}$ ("LP $11 \mathrm{~b}$ "). Further results for lower coupling values shown that all GDs present impulse-like PDFs. In Fig. 4 (b), for $-20 \mathrm{~dB} / 100 \mathrm{~m}$, the match between the simulated PDFs and the analytical PDF for strong coupling is good, even though the GDs have been normalized by different factors (Eq. (1)). Further results shown that the match between the simulated PDFs and the analytical PDF improves for higher coupling values. A similar match was obtained for 3 modes.

In a MDM system, in order to fully compensate for DMD and mode coupling, the MIMO equalizer must span a temporal memory at least as long as the system GD spread $\left(\tau_{6}-\tau_{1}\right)$. Fig. 5 shows the complementary cumulative distribution function (CCDF) of the normalized GD spread, $\operatorname{Pr}\left(\left(\tau_{6}-\tau_{1}\right) / \sigma_{g d}>p\right)$, obtained through simulation after $1000 \mathrm{~km}$ for different coupling values (averaging over 6000 different realizations). Fig. 5 shows that for $X T_{L P_{02}} \geq-30 \mathrm{~dB} / 100 \mathrm{~m}$ the CCDFs are very similar to the analytical approximation obtained for strong coupling [17] (dashed line). Conversely, for $X T_{L P_{02}}$ lower than $-30 \mathrm{~dB} / 100 \mathrm{~m}$ the normalized GD spread is significantly smaller than the normalized GD spread for strong coupling.

Combining this observation with the normalisation factor (Eq. (1)), we find that the required temporal equalizer memory length $\left(\Delta T_{E Q}\right)$ to span the channel memory with a given outage probability $p$ and for a given mode coupling strength is given by (in time units): 


$$
\begin{gathered}
\Delta T_{E Q} \cong U(p) \sigma_{g d}=U(p) \sqrt{2} \sigma_{\tau} L_{c}\left(e^{-L / L_{c}}+1 / L_{c}-1\right)^{1 / 2} \\
U(p)=x: \operatorname{Pr}\left\{\left(\tau_{6}-\tau_{1}\right) / \sigma_{g d} \leq x\right\}=p \\
\operatorname{Pr}\left\{\left(\tau_{6}-\tau_{1}\right) / \sigma_{g d} \leq x\right\}=-\int_{-\infty}^{+\infty} \partial F(t, y) /\left.\partial y\right|_{t=y+x} d y \\
-\partial F(t, y) / \partial y=6 ! /\left(\pi^{3} 2^{-15} \prod_{n=1}^{6} n !\right) \cdot \sum_{n=1}^{6} \operatorname{det}\left[\begin{array}{l}
I_{i+j-1}(x, y), i \neq n \\
y^{i+j-2} e^{-y^{2}}, i=n
\end{array}\right]_{i, j=1, \ldots, 6}
\end{gathered}
$$

where $U(p)$ can be calculated using (5) derived in [17], dashed line in Fig. 5. A $\Delta T_{E Q}$ from $4 \sigma_{g d}$ to $5 \sigma_{g d}$ is sufficient to span the channel memory with an outage probability from $10^{-4}$ to $10^{-6}$.

\section{Conclusions}

In this paper, we reported an investigation on the statistics of the group-delays in FMFs. The investigation shows that even for the FMFs in the literature presenting high coupling strength $(-7 \mathrm{~dB} / 100 \mathrm{~m})$, the performance is not accurately modelled by random unitary matrices (every $\sim 100 \mathrm{~m}$ ), which give a factor of 2 error. Furthermore, an analytical estimation for the maximum GD spread as a function of the coupling strength, was validated for FMFs guiding up to $6 \mathrm{LP}$ modes and for any coupling regime. Therefore, this analytical estimator is a valuable tool for the development of future FMF systems.

\section{Acknowledgments}

This work has been partially supported by the European Union (Grants 619732-INSPACE, 654809-HSPACE, 659950-INVENTION, and 627545-SOLAS), and by the EPSRC (Grant EP/L000091/1-PEACE). To access the research data supporting this publication, see http://dx.doi.org/10.17036/80b2ce0e-f835-4b02-b046c28909e71128.

\section{REFERENCES}

[1] K. Ho, et al., "Statistics of group delays in multimode fiber with strong mode coupling," J. Lightw. Technol., Vol. 29, no. 21, p. 3119 (2011).

[2] C. Antonelli, et al., "Stokes-space analysis of modal dispersion in fibers with multiple mode transmission," Opt. Express, Vol. 20, no. 11, p. 11718 (2012).

[3] F. Ferreira, et al., "Impact of Linear Mode Coupling on the Group Delay Spread in Few-Mode Fibers," Proc. OFC 2015, p. Tu2D.1, (2015).

[4] F. Ferreira, et al., "Few-Mode Fibre Group-Delays with Intermediate Coupling," in Proc. ECOC 2015, p. Th.1.6.1, (2015).

[5] S. Ö Arık, et al., "Delay Spread Reduction in Mode-Division Multiplexing: Mode Coupling Versus Delay Compensation," J. Lightw. Technol., vol. 33, no. 21, p. 4504 (2015).

[6] C. Poole, "Statistical treatment of polarization dispersion in single-mode fiber," Opt. Lett., Vol. 13, no. 8, p. 687 (1988).

[7] P. Wai, et al., "Polarization mode dispersion, decorrelation, and diffusion in optical fibers with randomly varying birefringence,” J. Lightw. Technol., vol. 14, no. 2, p. 148 (1996).

[8] S. Fan, et al., "Principal modes in multimode waveguides," Opt. Lett., Vol. 30, no. 2, p. 135 (2005).

[9] L. Grüner-Nielsen, et al., "Few Mode Transmission Fiber With Low DGD, Low Mode Coupling, and Low Loss," J. Lightw. Technol., Vol. 30, no. 23, p. 3693 (2012).

[10] T. Mori, et al., "Low DMD Four LP Mode Transmission Fiber for Wide-band WDM-MIMO System," Proc. OFC 2013, p. OTh3K.1 (2013).

[11] R. Ryf, et al., "Space-division multiplexed transmission over 4200-km 3-core microstructured fiber," Proc. OFC 2012, p. PDP5C.2 (2012).

[12] N. Fontaine, et al., "Experimental investigation of crosstalk accumulation in a ring-core fiber," Proc. PSSTMS 2013, p. TuC4.2 (2013).

[13] F. Ferreira, et al., "Nonlinear Semi-Analytical Model for Simulation of Few-Mode Fiber Transmission," Photon. Technol. Lett., Vol. 24, no. 4, p. 240 (2012).

[14] D. Marcuse, Theory of Dielectric Optical Waveguides. New York: Academic, 1974.

[15] F. Ferreira, et al., "Semi-analytical model for linear modal coupling in few-mode fiber transmission," Proc. ICTON 2012, p. Th.A1.5 (2012).

[16] F. Ferreira, et al., "Design of Few-Mode Fibers with M-modes and Low Differential Mode Delay," J. Lightw. Technol., Vol. 32, no. 3, p. 353 (2014).

[17] K. Ho, et al., "Delay-Spread Distribution for Multimode Fiber With Strong Mode Coupling," Photon. Technol. Lett., Vol. 24, no. 21, p. 1906 (2012). 